\title{
A Data Mining Approach to Identify Climatic Determinants of Dengue Fever Patterns in French Guiana
}

\author{
Claude Flamand*1, Mickael Fabregue ${ }^{2}$, Sandra Bringay ${ }^{3}$, Vanessa Ardillon ${ }^{4}$, Philippe \\ Quenel $^{5}$, Jean-Claude Desenclos ${ }^{6}$ and Maguelonne Teisseire ${ }^{7}$
}

${ }^{1}$ Institut Pasteur de la Guyane, Cayenne, French Guiana; 'LIRMM, CNRS, UMR 5506, Montpellier, France; ${ }^{3}$ MIAp Department, University Paul-Valery, Montpellier, France; ${ }^{4}$ Regional Epidemiology unit of Institut de Veille Sanitaire, Cayenne, French Guiana; ${ }^{5}$ Institut Pasteur de la Guyane, Cayenne, French Guiana; ${ }^{6}$ Institut de Veille Sanitaire, Paris, France; ${ }^{7}$ Laboratory Department of Information System, Irstea-TETIS, Montpellier, France

\section{Objective}

We used a data mining method based on sequential patterns extraction to identify local meteorological drivers of dengue fever epidemics in French Guiana.

\section{Introduction}

Epidemic dynamics of dengue fever are driven by complex interactions between hosts, vectors and viruses that are influenced by environmental and climatic factors [1]. The development of new methods to identify such specific characteristics becomes crucial to better understand and control spatiotemporal transmission. We concentrated our efforts on applying sequential pattern mining [2] to an epidemiological and meteorological dataset to identify potential drivers of dengue fever outbreaks.

\section{Methods}

Epidemiologic data on dengue fever were obtained for the period from 2006 to 2011. Climatic records including cumulative rainfall, minimum and maximum temperatures, sunstroke averages, wind strength, minimum and maximum relative humidity and global brilliance were obtained from meteorological stations of Météo France.

We applied contextual sequential pattern extraction techniques [3] to epidemiological and meteorological data to identify the most significant climatic factors for dengue fever, and we investigated the relevance of the extracted patterns for the epidemiological surveillance of dengue in French Guiana.

The methodology used involved 3 steps:

Step 1: The spatiotemporal resolution and the epidemiological contexts were defined;

- $\quad$ Step 2: The sequence preprocessing module transformed the raw data into sequences of events;

- $\quad$ Step 3: The sequential patterns extraction module extracted frequent sequences of events for each context.

\section{Results}

From the beginning of 2006 to April 2011, 39,587 clinical cases and 11,133 biologically confirmed cases were recorded in French Guiana. Global activity levels were strongly influenced by 3 major outbreaks during the study period. The duration of these epidemics varied from 38 to 41 weeks.

The beginning of an outbreak was frequently associated with a 4-week lag during which there was a strong increase in the minimum relative humidity, a decrease in the maximum temperature (after a peak observed 1 or 2 months before the start of the epidemic), and high but not too extreme levels of cumulative rainfall. The evolution of specific weather conditions was not associated with the epidemic peaks, where a predominance of the cumulative incidence occurred.

\section{Conclusions}

We demonstrated the use of contextual sequential patterns to better understand the determinants of the spatiotemporal spread of dengue fever in French Guiana. Compared with traditional models, such an approach can provide relevant insights that account for various temporal intervals and spatial units, and it is quite appropriate for comparing situations that can constrain analysts to multiply stratified analyses with traditional methods. Future works should integrate additional variables and explore the notion of neighborhood for extracting sequential patterns.

\section{Keywords}

Dengue fever; Data Mining; Infectious diseases; French Guiana; Meteorological factors

\section{Acknowledgments}

We are grateful to all of the collaborators involved in the surveillance system monitored by the Regional Epidemiology Unit. We wish to thank Meteo France for their help with the meteorological data collection.

\section{References}

[1] Racloz V, Ramsey R, Tong S, Hu W. Surveillance of dengue fever virus: a review of epidemiological models and early warning systems. PLoS Negl Trop Dis 2012;6(5):e1648.

[2] Agrawal, R., Srikant, R. Mining sequential patterns. In: Yu, P.S., Chen, A.S.P. (eds.) Eleventh International Conference on Data Engineering. IEEE Computer Society Press, 1995.

[3] Rabatel, J, Bringay, S, Poncelet, P. Contextual Sequential Pattern Mining. In: IEEE. 2010 IEEE International Conference on Data Mining Workshops. 2010:981-988.

\author{
*Claude Flamand \\ E-mail: cflamand@pasteur-cayenne.fr
}

